九州大学学術情報リポジトリ

Kyushu University Institutional Repository

\title{
On the Chaotic Nature of Turbulence Observed in Bench Mark Analysis of Nonlinear Plasma Simulation
}

Yagi, Masatoshi

Department of High Energy Engineering and Sciences, Interdisciplinary Graduate School of Engineering Sciences, Kyushu University

I. Itoh, Sanae

Department of High Energy Engineering and Sciences, Interdisciplinary Graduate School of Engineering Sciences, Kyushu University

Itoh, Kimitaka

National Institute of Fusion Science

Fukuyama, Atsushi

School of Engineering, Okayama University

https://doi.org/10.15017/17408

出版情報: 九州大学大学院総合理工学報告. 18 (2)，pp. 175-182，1996-09-01. 九州大学大学院総合理工 学研究科

バージョン：

権利関係 : 


\title{
On the Chaotic Nature of Turbulence Observed in Bench Mark Analysis of Nonlinear Plasma Simulation
}

\author{
Masatoshi Yagi*, Sanae-I. Itoh*, Kimitaka Itoh** \\ and Atsushi Fukuyama*** \\ (Received May 31, 1996)
}

\begin{abstract}
Bench mark test of the code for nonlinear simulation of plasma turbulence is performed. A new code describing four fields (B) is compared to the existing code (A) which treats three fields. Examining the results from two codes under the physically identical conditions, characteristics of the deviations are analyzed. It is found that the infinitesimally small initial noise, due to the cancelling, grows in accordance with the nonlinear development of turbulence mode. Interaction with an intrinsic nonlinearity of the system makes the noise grow, whose contribution becomes similar magnitude to the fluctuation itself of the results. The instantaneous deviation shows the chaotic characteristics. The spectrum analysis is made. These show the intrinsic nonlinearity of the plasma turbulence.
\end{abstract}

\section{Introduction}

Anomalous transport of high temperature plasma in toroidal devise is one of the key issues in the physics of plasmas. It is much larger than that caused by the binary collision of particles through Coulomb interactions and is considered to be caused from the fluctuations ${ }^{1)}$. Much work ${ }^{2-9)}$ has been done both theoretically and numerically. Several theoretical models have been proposed with respect to the driving mechanisms as well as the damping mechanisms. Recently new theoretical approach has been developed ${ }^{10)}$, which has predicted the nonlinear self-sustained turbulence in plasmas. In this theory, the strong turbulence is sustained by the nonlinear driving source and nonlinear damping effect. At the same time, the direct numerical simulations have been performed in order to reveal the quantitative feature of the plasma turbulence. Many of them have disregarded the electron nonlinearity, hence either the nonlinear driving in neglected ${ }^{11-14)}$ or the self-sustained turbulence has not been fully investigated. The direct simulation of Refs. ${ }^{15}{ }^{16)}$, could reveal the nonlinearly driven self-sustained turbulence of current-diffusive modes. Furthermore, the turbulence characteristic has been examined. ${ }^{15)}{ }^{16)}$ To strengthen the validity of the direct nonlinear simulation and to clarify the nature of current-diffusive mode, the bench mark analysis of the nonlinear simulation code is necessary.

In this paper, we report the results of bench mark analysis of nonlinear simulation codes. Two physically identical simulation codes are compared; one contains three field variables and the other has four fields. Comparing the results from two independent codes, we test the bench mark of four fields code. We find that the two physically identical codes give the same linear growth rate, nonlinear destabilization and similar saturation level. However, they did not show the identical temporal evolution under the physically identical situation. After some period, from the start simulation, the difference between the output of two codes becomes noticeable. It grows with the intrinsic nonlinear decorrelation time, which is predicted from

\footnotetext{
*Department of High Energy Engineering Science

${ }^{* *}$ National Institute for Fusion Science

${ }^{* * *}$ School of Engineering, Okayama University
} 
the basic equations. In the nonlinear saturation phase, the simulation results in both codes fluctuate in time around the mean values. Variances of the fluctuations become the order of the mean values. The difference between the results of two codes is originated from the very small but finite initial noises $\left(<10^{-8}\right)$. The relative difference stays constant in the phase of linear growth. The fluctuations in the simulation results show the typical chaotic nature in the nonlinear phase. This chaotic characteristics are originated from the nonlinearlity contained in the basic equations. Therefore, the analysis of this chaos reveals the aspect of the considered turbulence. In the following, we show the basic equations of nonlinear simulation codes and the conditions of the bench mark test in Section 2. The results of bench mark tests are reported and the analysis on the results are done in Section 3. The final section is devoted to the summary and discussions.

\section{Basic Equations of Current-Diffusive Turbulence}

We first show the basic equations which are used for the bench mark test. The current-diffusive interchange mode (CDIM) turbulence is considered. This turbulence is driven by the pressure gradient and is further destabilized by the electron nonlinearity .

We consider the cylindrical plasma immersed in a strong magnetic field in the $\mathrm{z}$-direction, $B_{0}$. The magnetic field has the shear in the radial direction as, $B_{\theta}=a B_{0} / q R$, where $a$ and $R$ the minor and major radii of the torus, $q$ is the safety factor. We consider that the mode is localized in the radial direction near the mode rational surface and the variable $x=r-r_{0}$ is used, where $r_{0}$ represents the location of the rational surface. The coordinates $(x, y, z)$ are taken such that $x$ is in the direction of pressure gradients, $z$ is that of magnetic field at $x=0$, and $y$ is that of perpendicular to and respectively. To describe CDIM, we employ the quasi-neutrality condition, the electron parallel momentum equation (which corresponds to the Ohm's law) and the pressure balance equations. One set of equations for three field analysis contains the total pressure balance equation and is given by ${ }^{17}$

$$
\begin{aligned}
& \frac{\partial}{\partial t} \nabla_{\perp}^{2} \phi=i k_{y} s x j-i \alpha k_{y} p-\left[\phi, \nabla_{\perp}^{2} \phi\right]+\mu_{\perp c} \nabla_{\perp}^{4} \phi, \\
& \frac{\partial}{\partial t} j=i k_{y} s x \phi-[\phi, j]+\lambda_{\perp c} \nabla_{\perp}^{2} j \\
& \frac{\partial}{\partial t} p=i k_{y} \phi-[\phi, p]+\chi_{\perp c} \nabla_{\perp}^{2} p,
\end{aligned}
$$

where $\phi$ is the electrostatic potential, $j$ is the current and $p$ is the total sum of electron pressure $p_{e}$ and ion pressure $p_{i}\left(p=p_{e}+p_{i}\right)$. Poisson's bracket, denoting the $\boldsymbol{E} \times \boldsymbol{B}$ nonlinearity, is defined as

$$
[\phi, A] \equiv(\partial \phi / \partial x)(\partial A / \partial y)-(\partial \phi / \partial y)(\partial A / \partial x)
$$

The normalization is employed as $t / \tau_{A \theta} \rightarrow t, r / \delta \rightarrow r, c \tau_{A \theta} \phi / B_{0} \delta^{2} \rightarrow \phi, 4 \pi r j / c B_{\theta} \rightarrow j, L_{p} p_{e} / \delta p_{0} \rightarrow p$, $\tau_{A \theta} \mu / n_{0} m_{i} \delta^{2} \rightarrow \mu, \tau_{A \theta} \lambda / \delta^{2} \rightarrow \lambda, \tau_{A \theta} \chi / \delta^{2} \rightarrow \chi ; \delta=c / \omega_{p e}, \tau_{A \theta}^{2}=4 \pi n_{0} m_{i} r^{2} / B_{\theta}^{2}$ and $L_{p}^{-1}=-d \ln p_{0} / d r$. The parameter $s$ denotes the magnetic shear, $s=r d \ln q / d r,\left[L_{s}^{-1}=s /(q R)\right], \mu_{\perp c}, \lambda_{\perp c}$, and $\chi_{\perp c}$ present the collisional viscosity, current diffusivity and thermal conductivity, respectively. In this system of equations, the ballooning parameter with $\alpha=-q^{2} R \beta^{\prime}$ with $\beta=8 \pi p_{0} / B_{0}^{2}$, the combination of the pressure gradient and the bad curvature, denotes the driving force. The Boussinesq approximation is used to derive these equations. The field quantities $(\phi, j, p)$ are invariant in the $z$-direction. 
The energy evolution of these equations is given as

$$
\frac{d E_{T}}{d t}=(\alpha+1)\left\langle\phi^{*} \frac{\partial \mathrm{p}}{\partial y}\right\rangle-\mu_{\perp c}\left\langle\left.\nabla_{\perp}^{2} \phi\right|^{2}\right\rangle-\lambda_{\perp c}\left\langle\left|\nabla_{\perp} j\right|^{2}\right\rangle-\chi_{\perp c}\left\langle\left|\nabla_{\perp}\right|^{2}\right\rangle
$$

where

$$
E_{T}=W_{\nabla \cdot \phi}+W_{j}+W_{p}
$$

with $W_{\nabla \phi}=\frac{1}{2}\left\langle\left|\nabla_{\perp} \phi\right|^{2}\right\rangle, W_{j}=\frac{1}{2}\left\langle|j|^{2}\right\rangle, W_{p}=\frac{1}{2}\left\langle|p|^{2}\right\rangle . \quad W_{\phi}$ corresponds to the electric field energy (and is almost equal to the kinetic energy of perpendicular ion motion), $W_{j}$ to the kinetic energy of the parallel electron motion, and $W_{p}$ to the electron internal energy. $\phi^{*}$ means the complex conjugate of $\phi$. The bracket \langle\rangle is defined as

$$
\langle f\rangle=\frac{1}{L_{x} L_{y} L_{z}} \int_{-L x / 2}^{L x / 2} d x \int_{0}^{L y} d y \int_{0}^{L z} d z f(x, y, z),
$$

where $L_{x}, L_{y}$ and $L_{z}$ are the system size of the simulation in the $x, y$ and $z$ directions, respectively. The power spectrum of $W_{\nabla \phi}\left(k_{y}, k_{z}\right), W_{j}\left(k_{y}, k_{z}\right), W_{p}\left(k_{y}, k_{z}\right)$, are defined as

$$
\begin{aligned}
& W_{\nabla \phi}\left(k_{y}, k_{z}\right)=\frac{1}{L_{x}} \int_{0}^{L x} d x \frac{1}{2}\left|\nabla_{\perp} \phi\left(x, k_{y}, k_{z}\right)\right|^{2}, \\
& W_{j}\left(k_{y}, k_{z}\right)=\frac{1}{L_{x}} \int_{0}^{L x} d x \frac{1}{2}\left|j\left(x, k_{y}, k_{z}\right)\right|^{2}, \\
& W_{p}\left(k_{y}, k_{z}\right)=\frac{1}{L_{x}} \int_{0}^{L x} d x \frac{1}{2}\left|p\left(x, k_{y}, k_{z}\right)\right|^{2},
\end{aligned}
$$

and $W_{T}\left(k_{y}, k_{z}\right)=W_{\nabla_{\phi}}\left(k_{y}, k_{z}\right)+W_{j}\left(k_{y}, k_{z}\right)+W_{p}\left(k_{y}, k_{z}\right)$.

Another set of equations contains four field, in which the electron pressure and ion pressure can change independently. The equation for the total pressure gradient (Eq. (3)) is replaced by two independent pressure gradients of electron and ions, i.e.,

$$
\begin{aligned}
& \frac{\partial}{\partial t} \nabla_{\perp}^{2} \phi=i k_{y} s x j-i \alpha_{e} k_{y} p_{e}-i \alpha_{i} k_{y} p_{i}-\left[\phi, \nabla_{\perp}^{2} \phi\right]+\mu_{\perp c} \nabla_{\perp}^{4} \phi, \\
& \frac{\partial}{\partial t} j=i k_{y} s x \phi-[\phi, j]+\lambda_{\perp c} \nabla_{\perp}^{2} p_{i}, \\
& \frac{\partial}{\partial t} p_{i}=i k_{y} \phi-\left[\phi, p_{i}\right]+\chi_{\perp c} \nabla_{\perp}^{2} p_{e}, \\
& \frac{\partial}{\partial t} p_{e}=i k_{y} \phi-\left[\phi, p_{e}\right]+\chi_{\perp c} \nabla_{\perp}^{2} p_{e},
\end{aligned}
$$

where $\alpha_{e, i}=-q^{2} R \beta_{e, i}^{\prime} . \quad$ Other notions are the same as for the set of Eqs.(1)-(3). If we set

$$
p_{e}=p_{i}=p, \alpha_{e}=\alpha_{i}=\frac{\alpha}{2}
$$

Eqs. (8)-(11) describes the physically identical case which is governed by Eqs. (1)-(3). Using these two set of equation, we performed the bench mark analysis of the code for Eqs.(8)-(11).

Before showing the simulation results, let us briefly discuss the characteristics of this system. These sets of equations contain the linear instability. For the parameters $s=\alpha=$ $0.5, \lambda_{\perp c}=0.01$ and $\mu_{\perp c}=\lambda_{\perp c}=0.2$, which are used in the simulation, we observe that the linear instability appears in the range of $0.2<k_{y}<0.9$ and the maximum linear growth rate is given as 


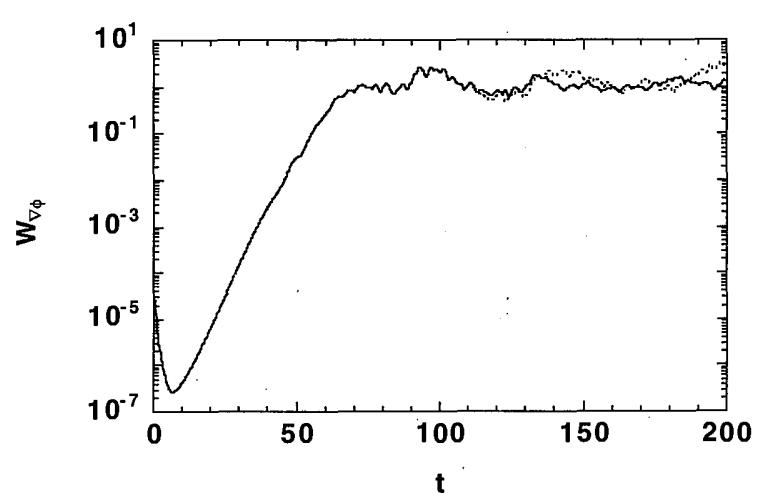

Fig. 1 (a)

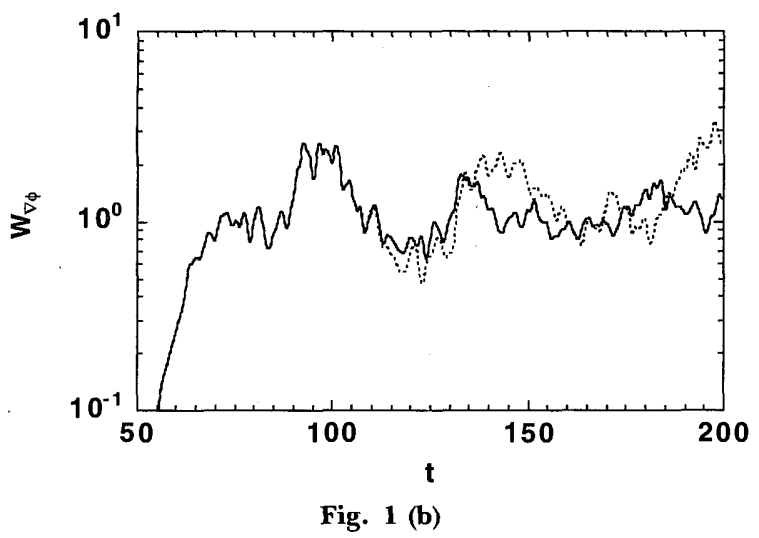

Fig. 1 Temporal evolutions of $W_{\nabla \phi}$ are drawn for the case of three field (code A) by the dashed line and for four field (code B) by the solid line (a). Physically identical two codes show the considerable deviation in the solutions after the nonlinear growth of the mode (for $t>120$ ). The expanded view is shown in. (b).
$\gamma_{L} \sim 0.17$ for $k_{y} \sim 0.5 . \quad$ In addition to the linear instability, this system is known to have the nonlinear instability. The nonlinear growth appears for the fluctuation level $W_{\nabla \phi}>10^{-3}$ for these parameters. And the nonlinear thermal diffusivity, $\chi_{N} \sim 3$ at the saturated stage has been obtained ${ }^{15), 16)}$.

\section{Nonlinear Simulation and the Bench Mark Results}

Nonlinear simulations are performed by directly solving Eqs. (1)-(3) and Eqs. (8)-(11) and the results are compared. The parameters $s=\alpha=0.5, \lambda_{\perp c}=0.01$ and $\mu_{\perp c}=\lambda_{\perp c}=$ 0.2 are employed, and Eq. (12) is used for the four field model. The slab geometry of the system size of $L_{x}=80$ in the $x$-direction and $L_{y}=6.4 \times 2 \pi$ in the $y$-direction is employed. The periodic condition is taken in the $y$-direction and $\mathrm{M}=64$ modes are taken in $k_{y}$ space $\left(k_{y, \text { min }}=10 / 64\right.$ and $\left.k_{y, \text { max }}=10\right)$. Let us call the code (A) for the case of solving. Eqs. (1) - (3) and the code (B) for solving Eqs. (8)-(11). Code (A) has three field for which the numerical resolution and accuracy have already been checked. For numerical resolution we carefully checked the dependence on the system size and confirmed the numerical accuracy ${ }^{16)}$. The convergence

study is also performed by changing the mode numbers, $\mathrm{M}^{17)}$.

Figure 1 (a) compares the time traces of $W_{\nabla \phi}$ of code (A) (dashed line) and code (B) (solid line). We observe that 1) for the period $t \leq 100$ the results from code (A) and code (B) agree well, however, 2) the difference becomes noticeable when $t \gtrsim 100$ and the deviation becomes appreciable for $t \geq 110$. The derivations from the temporal averaged values are seen to be chaotic at around $t \sim 200$, even though the averaged values of cases (A) and (B) are common. The expanded view of Fig. 1 (a) is shown in Fig. 1 (b). The deviations are seen more prominently and the variances becomes appreciable with respect to the averaged values. The similar behavior is also observed in $W_{p}(t)$ and $W_{j}(t)$.

In order to analyze these derivations, Fig. $\mathbf{2}$ shows their difference, that is,

$$
\delta W=W_{\nabla \phi}(\operatorname{code} A)-W_{\nabla \phi}(\operatorname{code} B) .
$$

In the initial phase, $0<t<40$, the exponential growth (with linear growth rate) of the derivation $\delta W$ is seen. Then the growth of $\delta W$ is associated with the change of sign (for $50<t<110$ ). Finally, the absolute value of deviation $\delta W$ saturates with its sign change (for $120<t$ ). (The negative value of $\delta W$ is not shown in the graph, because the plot is logarithmic.) From this graph, we see that the difference between the results of the two codes continues to grow from the beginning of the simulation, although it becomes noticeable at $t \gtrsim 100$. In order to understand 
the causes of the growth of $\delta W$, the relative derivation is shown in Fig. 3 by plotting the value of

$$
\varepsilon \equiv \frac{\left|W_{\nabla_{\phi}}(\operatorname{code} B)-W_{\nabla_{\phi}}(\operatorname{code} \mathrm{A})\right|}{\left|W_{\nabla_{\phi}}(\operatorname{code} B)+W_{\nabla_{\phi}}(\operatorname{code} \mathrm{A})\right|} .
$$

The relative derivation $\varepsilon$ does not change during the period of $0<t<40$. The relative difference starts to change its characteristics around $t \simeq 45$, and grows in the time period of $50<t<110$. The saturation at around 120 $<t$ are observed. Comparing the results of Fig. 2 and Fig. 3, we see that the initial noise is the origin of these deviations. During the period of $0<t<40$, the difference grows in accordance with the liner growth rate that the original set of equations have. Both the codes (A) and (B) precisely recover the linear growth rate, so that the relative difference remains unchanged. On the other hand, for $t>70$ the initial noise nonlinearly grows according to the nonlinear decorrelation rate of the system. Deviation saturates at around $t>120$ and the value becomes the order of averaged value,

$$
\varepsilon \sim 0.171
$$

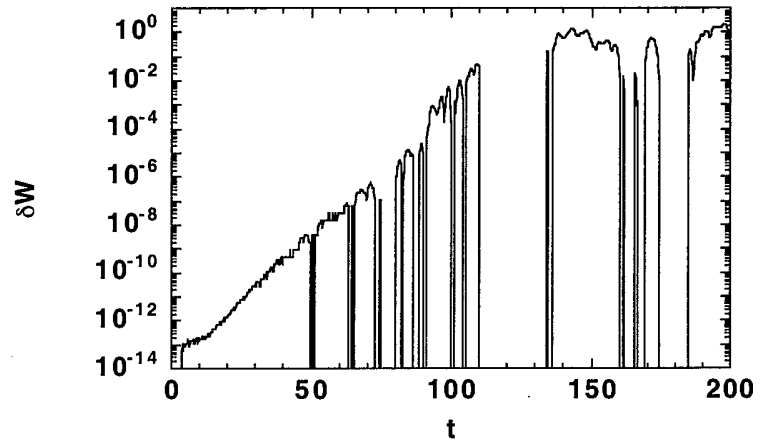

Fig. 2 The difference, $W_{\nabla \phi}($ code $\mathrm{A})-W_{\nabla \phi}($ code $\mathrm{B})$, is plotted against time.

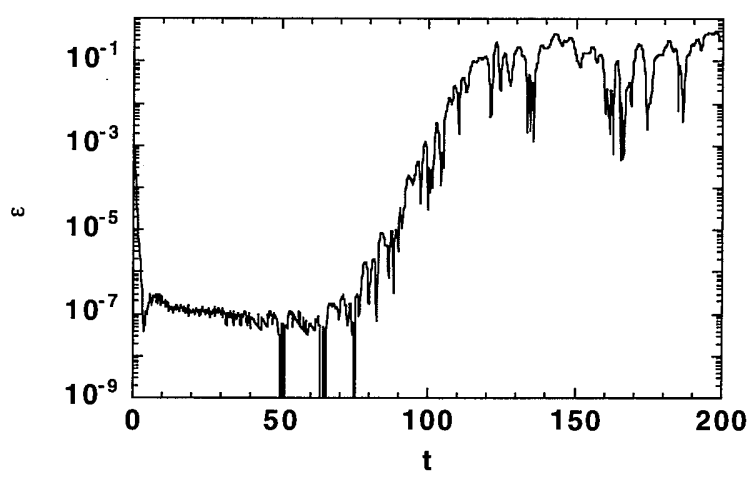

Fig. 3 The time trace of the relative deviation $\varepsilon \equiv \mid W_{\nabla_{\phi}}($ code $\mathrm{A})-W_{\nabla_{\phi}}(\operatorname{code} \mathrm{B})|/| W_{\nabla_{\phi}}(\operatorname{code} \mathrm{A})+W$ $\nabla_{\phi}($ code $\mathrm{B}) \mid$ is plotted. In the linear phase, the difference which is caused by the initial noise remains constant. The deviation grows associated with the nonlinear growth and saturation of the mode.

The nonlinear growth rate of the deviation $|\delta W|=\left|W_{\nabla \phi}(\operatorname{code} A)-W_{\nabla \phi}(\operatorname{code} B)\right|$ is estimated by fitting the data to the form of $\exp \left(\gamma_{a c} t\right)$. We obtain the value of

$$
\gamma_{a c} \sim 0.4 .
$$

In this system, the typical mode number of this turbulence is order of $k_{y} \sim 0.3$ and the nonlinear decorrelation rate of such a mode due to the nonlinear coupling is estimated to be $\gamma_{N L} \sim \chi_{N L} k_{y}^{2} \sim$ 0.3. This suggests that the initial noise due to the cancelling also grows under the nonlinear interactions of the turbulence mode. The initial noise itself grows in accordance with the linear growth rate, and the small difference in the initial nonlinear phase grows with the nonlinear decorrelation rate of the concerned mode. This reflects that the system has positive Lyapunov exponent under the observed turbulence level. Namely, the phase of the turbulence is also randomized due to the initial small noise.

Let us show the differences in $\mathrm{k}_{y}$-spectrum. In Fig. 4 (a) the $\mathrm{k}_{y}$-spectrum $W_{\nabla_{\phi}}\left(k_{y}\right)$ of code A (dashed lines) and of code B (solid lines) are compares for the times $t \simeq 10$ of and $t \simeq 150$. The difference is very small in the linear growth regime $(t \sim 10)$, but the discrepancy becomes appreciable in the nonlinearly developed phase $(t \sim 150)$. It is also noted that the difference

$$
\delta W_{\nabla \phi}\left(k_{y}\right) \equiv W_{\nabla \phi}\left(k_{y} ; \operatorname{code} A\right)-W_{\nabla \phi}\left(k_{y} ; \operatorname{code} B\right)
$$




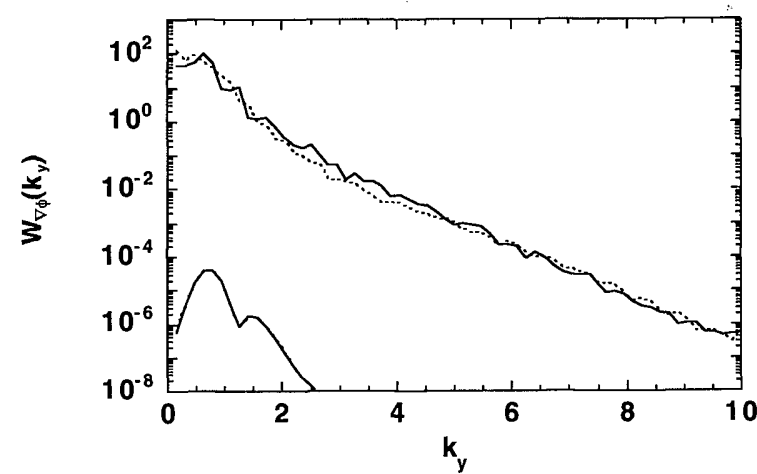

Fig. 4 (a)

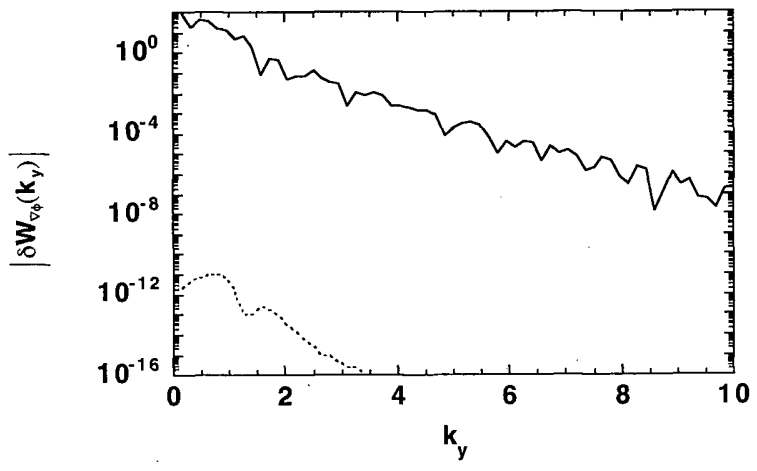

Fig. 4 (b)

Fig. 4 The $k_{y}$ spectrum of $\delta W_{\nabla \phi}\left(k_{y}\right)$ are plotted for $t \sim 10$ and $t \sim 150(\mathrm{a})$. The dashed lines are from code $A$ and the solid lines are from code $B$. The absolute values of the difference, $\mid W_{\nabla_{\phi}}\left(k_{y} ;\right.$ code $\left.\mathrm{A}\right)$ - $W_{\nabla_{\phi}}\left(k_{y} ;\right.$ code B) $\mid$, are plotted for $t \sim 10$ (broken line) and for 150 (solid line), respectively, in (b).

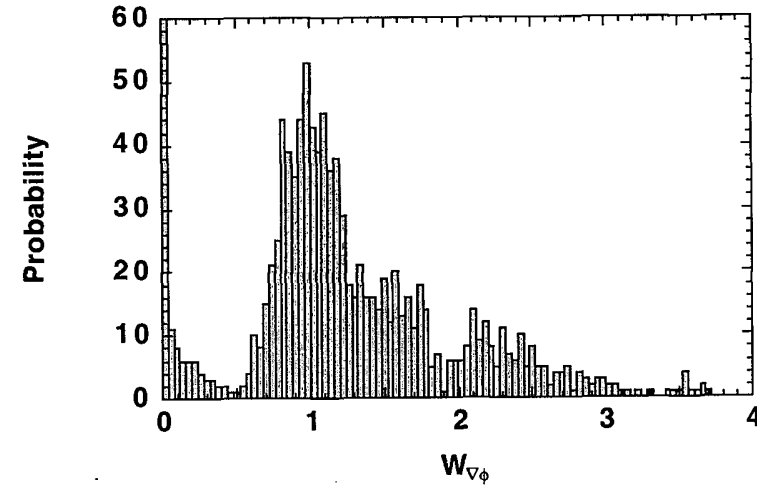

Fig. 5 The histogram of probability, that $W_{\nabla \phi}$ takes the value in the region, $(n-1) \delta<W_{\nabla_{\phi}}\left(t_{j}\right)<n \delta, \quad(n=$ $1,2, \cdots, 0.04)$ is shown. The total number of the data points, $t_{j}$, is 1261 . The fractal nature is seen.

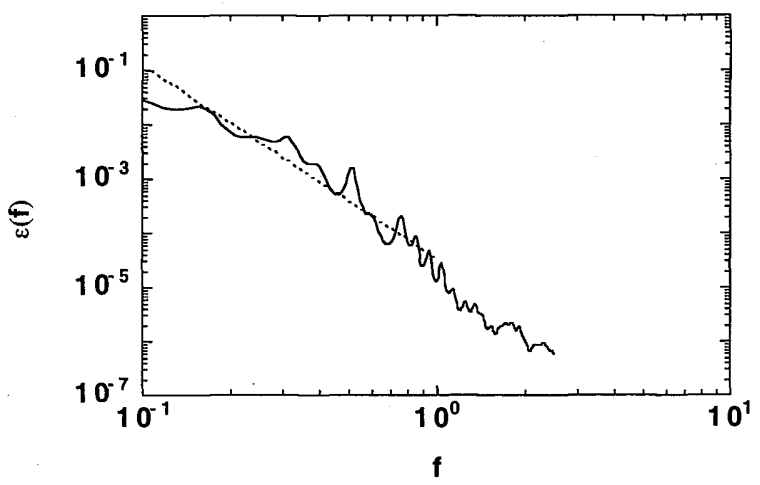

Fig. 6 The power spectrum of the relative deviation shown in Fig. 3 is plotted. The power law, which indi. cates the chaotic characteristics, is observed.

becomes either positive or negative, depending on the value of $k_{y}$. Small but finite difference $\delta W_{\nabla \phi}\left(k_{y}\right)$ evolves due to nonlinear interactions. The absolute values of the difference in $\mathrm{k}_{y}$-space, $\left|\delta W_{\nabla \phi}\left(k_{y}\right)\right|$, are plotted in Fig. 4 (b) for $t \sim 10$ and $t \sim 150 . \quad$ In the nonlinearly developed phase, the initial noise also develops to large difference widely in the $k_{y}$-space. From these observations, we see that the instantaneous deviation $\delta W / W$ reaches to the value of the order of unity. Namely, difference of the nonlinear simulation results, which is caused by the infinitesimally small noise at the initial phase, become the order of the time averaged value the solution.

The histogram in Fig. 5 shows the probability distribution of $W_{\nabla \phi}(t)$ in the simulation. The number of the data $\delta W_{\nabla \phi}\left(t_{j}\right)$ points in the simulation steps, $t_{j}$, whose value drops in the region $(n-1) \delta<\delta W_{\nabla \phi}\left(t_{j}\right)<n \delta,(n=1,2 \cdots, \delta=0.04)$, are plotted. The time series data of $W$ ${ }_{\nabla \phi}\left(t_{j} ; \operatorname{code} B\right)$ contains 1261 points and their distribution is plotted. (Data close to zero, $W_{\nabla \phi}\left(t_{j}\right)$ $<0.04$, shows the overscale; however, these data are originated from the linear growth phase $t$ <40.) We are interested in the data of fully nonlinearly developed phase, $W_{\nabla \phi}\left(t_{j}\right)>0.5$. The number along the vertical axis corresponds to the probability that the $W_{\nabla \phi}\left(t_{j}\right.$; $\left.\operatorname{code} B\right)$ takes the value between $(n-1) \delta$ and $n \delta$. It is seen that the averaged value is $\sim 1.42(t>70)$. However, the deviation from the mean value is large as

$$
\frac{\sqrt{\{W-\langle W\rangle\}^{2}}}{\langle W\rangle} \sim O(1) \text {. }
$$


The distribution is not Gaussian and has the tail component. It can be fitted to the power distribution. This analysis shows the characteristics of intrinsic nonlinearlity which the basic equations have. The large value of the dispersion shown like in Fig. $\mathbf{5}$ corresponds to the fact that the relative deviation of two solutions by code (A) and (B) becomes $\sim O(1)$ after the initial memory of phase is lost.

Power spectrum of $\varepsilon(t)=\left|W_{\nabla \phi}(\operatorname{code} A)-W_{\nabla \phi}(\operatorname{code} B)\right| /\left|W_{\nabla \phi}(\operatorname{code} A)+W_{\nabla \phi}(\operatorname{code} B)\right|$ is plotted in Fig. 6. Power Spectrum $\varepsilon(f)$ is evaluated from the data $\varepsilon(t)$, by use of Maximum Entropy Method. If it is fitted in a form of $f^{-\eta}$ in the range of $0.1<f<1$, the spectrum is fitted to $f^{-3.58}$. The value of $3.58(>3)$ is not accurate enough, however, the power spectrum of the relative derivation shows the fractal characteristic and indicates the chaos nature of the fluctuation.

\section{Summary and Discussions}

In summary, we performed the bench mark test of a new nonlinear simulation code of four field (code B), comparing it with the results from the code of three field (code A). It is found that two different codes reproduce the same linear growth rate, nonlinear growth and saturation level. However, the two codes do not show the same temporal evolution even if the model equations are physically identical (Fig. 1). This is because the infinitesimally small noise at the initial loading due to the cancelling of each code grows in the system of nonlinear equations and the noise itself grows nonlinearly. The information of the phase is lost. The difference of the two codes reflects the intrinsic chaos in the turbulence which is described by the set of equations.

The derivation of code $B$ 's solution from that of code $A$ 's grows linearly and nonlinearly in accordance with the linear and nonlinear solutions of $(A)$ and $(B)$ (Fig. 2). The relative deviation stays constant during the linear phase, grows nonlinearly and saturates in the nonlinear phase. The deviation becomes order of the mean value of the solution and shows the chaotic characteristics (Fig. 3). The deviations in $k_{y}$ space and in frequency range are analyzed and the fractal (chaotic) characteristics are extracted (Fig. 4, 6). From the data of time series, we find that the instantaneous deviation from the mean value becomes of the order of unity and that the deviations show the chaotic nature in the nonlinearly developed phase (Fig. 5), where the initial memory of the phase is lost.

The noise installed at the initial loading is inevitable in this kind of nonlinear simulations. Therefore it is very important to know how this noise affects the simulational results. We explored some of them. The analyses made in this text correspond to the case that an artificial passive noise is added to the basic nonlinear equations. Analyses using the passive noise may contribute to a test probing method to understand the simulational results of the turbulence of concern. In order to analyze the nonlinear development of the mode in the presence of noise, the active noise (called noise pumping) has been introduced. The comparison study of the cases of the active noise and the passive noise may elucidate an aspect of nonlinear dynamics of the mode. These are open for future analysis.

\section{Acknowledgment}

This work is partly supported by Grant-in-Aid for Encouragement of Young Scientists, Interdisciplinary Graduate School of Engineering Sciences of Kyushu University, to which we would like to acknowledge.

This work is also partly supported by the Grant-in-Aid for Scientific Research of The Ministry of Education, Science and Culture, Japan, and by the collaboration program of Advanced Fusion Research Center of Kyushu University. 


\section{Reference}

1) P. C. Liewer, Nucl. Fusion 25, 543 (1985).

2) B. B. Kadomtsev and V. I. Petviashvili, Sov. Phys. JETP 17, 403 (1963).

3) W. Horton, Phys. Rep. 192 (1-3), p.1-177 (1990).

4) B. A. Carreras, L. Garcia and P. H. Diamond, Phys. Fluids 29, 3291 (1986).

5) S. P. Hirshman and K. Molvig, Phys. Rev. Lett. 42, 648 (1979).

6) R. D. Sydora, J. N. Leboeuf and T. Tajima, Phys. Fluids s, 528 (1985).

7) M. Wakatani and A. Hasegawa, Phys. Rev. Lett. 59, 1581 (1987).

8) B. D. Scott, Phys Fluids B4, 2468 (1992).

9) B. A. Carreras, V. E. Lynch, L. Garcia and P. H. Diamond, Phys. Fluids B5, 1491 (1993).

10) K. Itoh, S.-I. Itoh and A. Fukuyama, Phys. Rev. Lett. 49, 1408 (1992); K. Itoh, S.-I. Itoh, A. Fukuyama, M. Yagi and M. Azumi, Proceedings of 14th International Conference on Plasma Phys. Contr. Nucl. Fusion Research, Wurzburg, 1992, (International Atomic Energy Agency, Vienna, 1993), Vol.2, p.381; K. Itoh, S.-I. Itoh, A. Fukuyama, M. Yagi and M. Azumi, Plasma Phys. contr. Fusion 36, 261 (1994); 36, 279 (1994) ; 36, 1385 (1994) ; 36, 1501 (1994).

11) W. Horton, R. D. Estes and D. Biskamp, Plasma Physics 22, 663 (1980).

12) S. Hamaguchi and W. Horton, Phys. Fluids B2, 1833 (1990).

13) R. E. Waltz, Phys. Fluids 29, 3684 (1986).

14) AIP Conference Proceedings 284 U.S.-Japan Workshop on Ion Temperature Gradient-Driven Turbulent Transport, Austin $T X, 1993$, edited by W. Horton, M. Wakatani and A. Wootton (American Institute of Physics, New York, 1993).

15) M. Yagi, S.-I. Itoh, K. Itoh, A. Fukuyama and M. Azumi, Phys. Plasmas 2, 4140 (1995).

16) M. Yagi, Journal of Plasma and Fusion Research 71, 1123 (1995).

17) M. Yagi , K. Itoh, S.-I. Itoh, A. Fukuyama and.M. Azumi, Phys. Fluids B5, 3702 (1993), J. Phys. Soc. Jpn. 63, 10 (1994). 